\title{
Some aspects of using it-systems for logistics and cargo transportation
}

\author{
Olga Rudakova ${ }^{1}$, Lidia Terekhova ${ }^{2}$, Aleksandr Aleksakhin ${ }^{1 *}$, Nataliya Pianova ${ }^{1}$ \\ ${ }^{1}$ Russian Presidential Academy of National Economy and Public Administration (Central Russian \\ Institute of Management), 5A Pobedy Boulevard, Oryol, 302028, Russia \\ ${ }^{2}$ Oryol State University named after I. S. Turgenev, 95 Komsomolskaya str., Oryol, 302026, Russia
}

\begin{abstract}
Modern management of transport systems is characterized by the use of special automated and information systems. Along with them, digital technologies are gradually being used to bring the model of transport system management to a qualitatively new level. The use of multifunctional systems elevates the development of digital and information technologies of vehicle control.
\end{abstract}

Nowadays, IT systems are an integral part in the processes of managing the delivery of various goods, warehouse operations, or the activities of an organization. The ability to use these systems allows you to make fewer mistakes and reduce the role of a person in this work, and also contribute to increasing the productivity of not only the company itself, but also partners in the supply chain. IT systems are innovations that are constantly changing and require detailed study. It is very important to use the data correctly for the most productive implementation of the tasks set.

Information technology is a complex of interrelated scientific, technological, and engineering disciplines that study the issues and methods for the effective work organization of people engaged in processing and storing information; methods of organization and interaction with people and production equipment, their practical applications, as well as related social, economic, and cultural problems. Their use in the transport sector requires large financial and labour costs, but the result significantly simplifies and stabilizes the management capabilities of transport systems.

In recent years, modern vehicle management technologies have become increasingly important in the world. Such technologies include information systems that perform the function of transport control or management support. The information system includes a number of established principles and methods that ensure maximum labour productivity at minimal costs.

Due to the growth of cities, the current transport system can no longer cope with its functions. Information and transport technologies simplify the possibility of direct interaction with all parts of the system. To achieve this goal, a number of special mechanisms have been created. They are video cameras, traffic detectors, online timetables, computerization of transport management, electronic payment services for transportation.

\footnotetext{
${ }^{*}$ Corresponding author: aleksakhinan@yandex.ru
} 
All elements for intelligent management of transport systems simplify the development of other spheres in the society, ensure the emergence of new market relations, and increase the level of the population's possible needs (including the transport system). Digital transport technologies bring the commercial component to a new level, as it allows you to recognize the malfunction of a vehicle earlier or to conduct a qualitative statistical analysis of the transport system activity.

Even at the end of the 20th century, scientists began to discuss the idea of implementing intelligent transport systems due to its relevance. Traffic has both economic and social significance, people believed that with the introduction of ITS, it would be possible to rationalize the organization of traffic, to develop roads without the construction of new highways. The best scientists and specialists in the transport field and representatives of various other fields took up the development of the most optimal algorithms for the implementation and development of ITS. The introduction of innovative ITS technologies is primarily based on the modernization and radical rethinking of existing transport infrastructure processes. With the large scale of the transport system and a wide variety of technologies, this process is not able to cover all subsystems and elements at once. Therefore, this technology should be staged, only over time affecting an increasing number of life spheres.

Innovative transport technologies have a special impact on the technological and social sphere. Thus, the changes set up the society for a new vector of development, for the transition to modern technologies and a new life. However, not all the principles of transport system management are clear to the public. People have to get used to the new elements of commercial and social management.

Changes in the management technologies of transport systems affect more and more elements every year. This is especially seen in the following components:

- modes of transportation (new types of vehicles will not be familiar to ordinary users);

- $\quad$ automated methods of vehicle management;

- improvement of the transport movement system (traffic control in the city, comprehensive transformation of the transport system);

- new environmental principles (ecological fuel, transport means that do not pollute nature).

Ensuring the normal adaptation of society is also an important goal of the transport system development. As a result, a multiplatform should be created that collects and analyzes data on the transport situation in the city. The decision-making process should take a minimum of time so that the final user can purposefully manage part of the traffic flows network. This is a particularly important step in the development of modern transport system management technologies.

Synergy with modern ICT and automation tools with transport infrastructure, consumers and vehicles is aimed at increasing the safety and quality efficiency of transport processes. High-tech tools for managing the transport system are being constantly invented. For example, nowadays, various types of radio communication are increasingly used, which can extend both over short and long distances.

Innovative developments in the field of embedded systems technology promote the use of operating systems in real time and enable the use of high-level software applications in the field of artificial intelligence. Information technologies are also used to increase the power of processors in embedded computer systems, as well as to improve compatibility with personal computers making it possible to reuse code and transfer the most intelligent services.

The main advantages of modern intelligent technologies are:

- huge functionality that ensures continuity and constant monitoring of transport systems;

convenience, easy operation of tools (navigators, special video cameras); 
- $\quad$ informativeness (special tools allow users to get information while in transport).

Modern informatization of transport systems is becoming the basis for their further development. At the moment, a huge number of companies around the world are engaged in the creation and manufacture of information systems. The improvement of such systems is also an important task for modern society. For example, the German railways have created an integrated information system that provides an increased level of passenger service. A number of digital platforms are also being created. This is done by such companies as General Electric (Prefix), Siemens (Mind sphere), MAN (Rio), Airbus (Sky wise), Boeing (Analytics) and others. The platforms allow high-quality diagnostics, data collection and analysis to assess the state of the transport system. Moreover, modern manufacturers of transport tools are switching to a service model, offering consumers not one service, but a whole range of those ones.

Another important goal is to duplicate not only modern vehicles, but also more complex systems, such as the railway network. The Russian Railways company is actively moving in this direction, where, after a number of projects, a high-quality system for servicing trains of the Lastochka series was created.

The general trend, especially in the field of transport, is the use of neural networks, artificial intelligence systems and machine data analysis. These technologies make it possible to transfer processes made by humans to machines for their execution. For example, they can analyze video images, find objects that may pose a danger. Today, video analysis technologies are in demand for monitoring the degree of the driver's fatigue and his behavior while driving (eating, smoking), the use of a seat belt, the use of mobile phones. In addition, they help to monitor the number of passengers in a taxi, the identity of the driver. An integrated breathalyzer system already exists in the automotive market. Automation makes it possible to transfer many analytical works to a computer. Man continues to bear the burden of decision-making, but at some levels this stage is also provided by machines. Therefore, automatic decision-making can also be called a trend of future development.

As for the logistics of transport, it is a field of knowledge about the organized delivery of goods or passengers from one place to another in the shortest possible time and with minimal costs, provided that the delivered cargo is integral and safe. It is the use of computer IT and IP, logistics of cargo delivery that has become the main form for organizing the goods transportation in the technologically highly competitive market of transport services.

There are many IT systems designed to optimize processes and transition from paper work to digital work. Let us consider some of them.

The main task of the transport and logistics system is to meet the needs of users. Undoubtedly, the transport and logistics system implies the freedom of choice among consumers. This system includes several participants, whose activities should be coordinated and focused on achieving results while meeting the needs of service consumers and all participants in this system as much as possible.

Nowadays, we can distinguish the following IT tools for logistics. TMS helps to increase the quality characteristics of the cargo delivery process, manage the fleet of vehicles and optimize the supply chain. Systems can also provide other functions. For example, online monitoring of cargo delivery, coordinated creation and management of delivery schedules, tender purchases of various cargo shipments, and etc.

The warehouse management system (TSM - time slot management) ensures timely delivery. They allow you to plan and manage the schedule or time for vehicles arrival at the warehouse for loading and unloading. TSM is very convenient for those who work on a pick-up basis. So, a time gap is created for the customer, where he receives a notification about the exact date and time of shipment. The TSM system plays a very important role for companies with heavy cargo turnover: 
- for those who have any restrictions in the loading areas (the system creates time intervals that optimize the reception of cargo and shipment of goods);

- $\quad$ for companies with different business models of organization and different loading and unloading processes.

WMS is Warehouse management system. This system includes any technologies that involve automation of warehouse operations and their maximum digital integration with other business processes. The use of WMS is rational only for large companies.

Videotrans is an information system developed in Belgium and designed for the selection of cargo transportation, as well as for informing transport companies. This system allows the car company to receive certificates and enter information about the availability of vehicles or goods for delivery in their use.

Gonrand is an information system for the selection of cargo transportation. This system is designed to collect data on the availability of cargo. The software allows you to group cargo by various attributes and provide information about its shipment, information about the consignee, the registration number of the vehicle, the customer of the goods, the unit code and the amount of shipments.

Espace Cat is a French information system for optimizing cargo transportation. This system transmits information to the user about the goods being transported and creates diagrams of their location in the vehicle in the form of three-dimensional graphs. Space Cat software is simple and convenient, easily optimized to the needs of users.

CTC is a Swiss computer system for the selection of cargo transportation. It is able to inform about the availability of various goods, about the types of delivery vehicles, rational traffic patterns, contains the addresses of transport companies that currently have free rolling stock, etc.

BKS is another system for selecting cargo transportation. The company provides participants with timely payment for cargo transportation, even if the customer did not make a timely payment. This increases the attractiveness of the interaction and thus increases the reach of the consumer market.

FMS is Fleet management systems. Currently, there are a lot of proposals for fleet management systems. However, most of them are limited only to maintaining travel documentation. As a rule, large industrial enterprises need high-quality accounting of fuel and lubricants in sections by workshops, teams, and convoys. When taking into account itinerary, repair sheets, orders, the amount of accumulated data can be an impressive array.

MRO-system (Maintenance, Repair and Overhaul) is a vehicle repair and maintenance management system. The MRO system is used in aviation, urban and railway transport, shipbuilding, and the armed forces.

Recently, RFID technologies have become very popular in the logistics and accounting system of vehicles.

Container Terminal Vision (CV) is an information system that allows you to manage the processes of receiving containers arriving by various modes of transport. Through the RF terminals, tasks are transmitted to employees and technical means for moving the container to a given location. During shipment, the information system identifies each container and issues a task for selecting and moving the desired container. RF terminals and wireless networks are used to collect and transmit information in real time.

The ConsID CTMS system is designed to automate the management of the container terminal and all operations with containers and cargo on its territory. ConsID CTMS manages the use of re-loading equipment, vehicles and maintenance personnel, and registers container movements. All operations are performed using mobile data processing terminals, from which information is transmitted to the system. With the help of RFID identification technologies, the speed of data entry while selecting container processing tasks increases. This technology saves the operator from the monotonous manual input of container numbers. 
Thus, IT systems of logistics and cargo transportation are the main tools to carry out modernization in the transport sector. Hundreds of operations that took a long time to complete were simplified. These systems allow you to work out different planning schemes with better results, as well as to understand the possibilities of the decisions taken. It is very important to develop these systems to ensure the efficient operation of cargo transportation and work with them.

I would like to note that today more and more interest among market players is attracted by the robotization of intra-warehouse logistics, due to the fact that this dramatically contributes to improving productivity, service and accuracy. However, it is very difficult to implement this idea at the moment, because most companies are still not ready for robotics and at the same time use cheap labour. Nevertheless, the robotization of systems is a necessary condition of the modern world, where systems are becoming more and more complex, and operations are performed many times faster and more accurately.

The importance of information and digital technologies in the modern world is difficult to overestimate. Its main goal is to transform and combine different technologies into a single system of information technology environment. The use of such technologies is an important factor in the future development of global transport systems. In the near future, this will lead to the complete informatization of transport and the creation of its unified system.

\section{References}

1. V. Akberdina, A. Kalinina, A.Vlasov The transformation stages of the industrial complex in Russia under the conditions of the economy digitalization , Problems and prospects of management, №.16(4), pp. 201-211. (2018.) -

URL: https://elibrary.ru/item.asp?id=38621481.

2. D.R. Abdusheva, A.O. Merenkov, A.A. Stepanov Terms-structure of the marketing system digital transport and logistics in the management of competitiveness, Management, №3. pp. 21-27, (2018) -

URL: https://elibrary.ru/item.asp?id $=\% 2036290652$

3. T.V. Avdeenko., A.A Aletdinova Digitalization of the economy on the basis of improving expert knowledge management systems, Scientific and technical reports of Saint Petersburg State Technical University. Economic science, №10, pp. 47-55 (2017) -. URL: https://elibrary.ru/item.asp?id=\%2028794920

4. D.M. Zozulya Digitalization of the Russian economy and Industry 4.0: challenges and prospects, Issues of innovative economy, №1, pp. 1-14, (2018) - URL: https://elibrary.ru/item.asp?id=\%2032809291

5. N.V. Emelyanov Digitalization of the economy as a factor in ensuring national security of Russia, Economic growth: problems, patterns, and prospects, №5, pp. 89-93 (2019) -URL: https://elibrary.ru/item.asp?id=\%2036797919 